\title{
Comparación de la penetración de iones cloruro en los pilotes de hormigón armado de la Terminal de Cruceros enla bahía de la Habana, Cuba
}

\author{
A. Castañeda ${ }^{1 *}$, C. Valdés ${ }^{1}$, J. Viña ${ }^{1}$, U. Verdecia ${ }^{2}$, F. Corvo ${ }^{3}$, R. Marrero ${ }^{4}$ \\ *Autor de Contacto: abel.castaneda@cnic.edu.cu
}

${ }^{1}$ Laboratorio de Protección de Materiales. Dirección de Investigación, Desarrollo e Innovación. Centro Nacional de Investigaciones Científicas. Ave. 25, Esquina 158, Cubanacán, Playa. La Habana, Cuba.

${ }^{2}$ Empresa Nacional de Investigaciones Aplicadas. Calle 100, No. 9107, entre Vento y 10 Altahabana, Boyeros. La Habana, Cuba.

${ }^{3}$ Centro de Estudios de Corrosión. Universidad Autónoma de Campeche. Av. Héroe de Nacozari, No. 480 Campus VI de Investigación. San Francisco de Campeche, Campeche. México.

${ }^{4}$ Grupo de Investigación en Corrosión e Ingeniería de Materiales. Facultad de Ingeniería Química. Universidad Tecnológica de La Habana. Calle 114 No. 11901 entre Ciclo vía y Rotonda, Marianao, La Habana, Cuba.

\section{RESUMEN}

El trabajo se basó,en conocer el mecanismo de penetración de iones cloruroen pilotes de hormigón armado, expuestos a inmersión en agua de mar y su comparación con otros hormigones sometidos a diferentes condiciones de exposición. De esta manera, se procedió a demostrar, cómo influyó el comportamiento de la penetración de iones cloruro en la corrosión de los aceros de refuerzo en dependencia de la condición de exposición a las cuales fueron sometidos los hormigones.El comportamiento de la penetración de iones cloruro en los pilotes, en comparación con otros tipos de hormigones expuestos a la condición de exposición a la atmósfera bajo condiciones de muy elevada y extrema agresividad corrosiva de la atmósfera,resultó totalmente diferente.

Palabras clave: pilotes; cloruro; hormigón; corrosión; aceros de refuerzo.

\begin{abstract}
The work was based on understanding the mechanism of chloride ion penetration in reinforced concrete piles exposed to seawater immersion and its comparison with other concretes subjected to different exposure conditions. In this way, we proceeded to demonstrate how the behavior of chloride ion penetration influenced the corrosion of the reinforcement steels depending on the exposure conditions to which the concretes were subjected. The penetration behavior of chloride ions in the piles compared to other types of concrete exposed to the condition of exposure to the atmosphere under conditions of very high and extreme aggressivity corrosion of the atmospherewas totally different.
\end{abstract}

Key words: piles; chloride; concrete; corrosion; reinforcement steel.

\section{RESUMO}

O trabalhofoibaseado no conhecimento do mecanismo de penetração de íonscloretoem estacas de concreto armado expostas à imersãonaágua do mar e suacomparaçãocomoutros concretos sujeitos a diferentes condições de exposição.Desta forma, procedemos para demonstrar cómo o comportamento da penetração de íonscloretoinfluenciou a corrosão dos aços de reforço, dependendo das condições de exposiçãoàsquais os concretos foramsubmetidos.O comportamento de penetração de íonscloretonasestacasfoi totalmente diferente emcomparaçãocomoutros tipos de concreto expostos à condição de exposição à atmosfera sobcondições de muito alta e extrema agressividade corrosiva da atmosfera.

Palavras-chave: estacas; cloreto; concreto; corrosão; aços de reforço. 


\section{INTRODUCCIÓN}

El comportamiento de la penetración de iones cloruro, ha sido muy estudiado en los elementos constructivos que componen las estructuras de hormigón armado intensamente deterioradas y de forma anticipada a causa del fenómeno de la corrosión atmosférica del acero de refuerzo (Helene et al, 2003; El Fattah, et al 2018). Otros estudios en probetas de hormigón simple y armado, han sido ejecutados antes de proceder con las labores de construcción. (Troconis et al, 2007; Meira et al, 2007). Todos,con el fin de obtener criterios por durabilidad que permitan garantizar plazos elevados de vida útil, a partir de los trabajos de reparación y antes de proceder con las labores de construcción. Los elementos y las probetas han estado sometidos de forma directa al impacto fuerte del ambiente agresivo en ciudades costeras de elevado potencial constructivo con climas subtropical y tropical de Cuba y a nivel mundial, principalmente en América Latina. Valores elevados de humedad relativa, acompañado de variaciones de la temperatura y deposiciones elevadas de sales de iones cloruro, garantizan las categorías de elevada (C4), muy elevada (C5) y extrema (CX) de agresividad corrosiva de la atmósfera para el acero al carbono, bien definidas en la normativa de la especialidad de corrosión atmosférica (ISO-9223:2012). Como bien se conoce, la atmósfera por su gran extensión, es el medio natural al cual se encuentran expuestas la gran mayoría de las estructuras y no solo las de hormigón armado, sino también las construidas con los materiales metálicos más usados en la industria de la construcción como son los aceros al carbono y galvanizado (Castañeda, et al 2013).

Diversas investigaciones han demostrado también el comportamiento de la penetración de iones cloruro bajo condiciones de ensayos acelerados (Angst, et al 2009). Las probetas fueron colocadas en cámaras salinas. Lo que sucede realmente es que, las condiciones de estos ensayos acelerados no simulan los procesos transitorios de la atmósfera como la formación y desarrollo del aerosol marino y los regímenes de humedad relativa y temperatura. La formación de las olas debido a la influencia del viento, hace que el aerosol marino originado, transporte las sales de iones cloruro, que tienen como fuente de procedencia el mar, antes de ser impactadas y depositadas en las superficies de las estructuras o probetas (Meira, et al 2008).Con el propósito de obtener una respuesta científica más exacta y precisa, sería más conveniente someter las probetas de hormigón simple o armado a ensayos acelerados bajo condiciones reales de exposición a la atmósfera a partir de la aplicación de un spray salino de $\mathrm{NaCl}$ al 3\% (Castañeda, et al 2003).

Por otra lado, esta herramienta ingenieril importante de evaluación de la penetración de iones cloruro, que a partir de su comportamiento conduce a la selección adecuada de los esquemas de trabajos de mantenimiento y reparación a que serán sometidas la estructuras con un intenso deterioro o no, ha sido estudiada también bajo condiciones de laboratorio en probetas inmersas totalmente en disoluciones salinas de $\mathrm{NaCl}$ entre 3 y 5\%. De esta forma, se simula la penetración de las sales de iones cloruro hacia diferentes profundidades en el hormigón expuesto a la condición de exposición de inmersión completa en el agua de mar (Castañeda, et al 2005).

La concentración crítica de iones cloruro libres o solubles, que inicia y desarrolla la corrosión atmosférica partiendo de la ruptura de la capa pasiva formada en el acero de refuerzo embebido en el hormigón armado, ha resultado polémico a nivel mundial (Angst, et al, 2011; Pradhan, et al 2011; Xianming, et al 2012). Cada país tiene sus especificaciones en la tecnología del hormigón a la hora de su dosificación, elaboración y colocación a pie de obra. Por otro lado, influye también las diferencias en los tipos de cementosy áridos a usar a la hora de elaborar el hormigón.

De manera general, pocos estudios han sido ejecutados basados en evaluar la penetración de iones cloruro en elementos de hormigón armado expuestos a la condición de exposición de inmersión total en agua de mar en una bahía. El trabajo tiene como objetivos, conocer el mecanismo de penetración de iones cloruro en los pilotes de hormigón armado expuestos totalmente a inmersión en agua de mar, que sostienen las losas armadas de la Terminal de Cruceros de la bahía de la 
Habana, Cuba y su comparación con otros tipos de hormigones sometidos a otras condiciones de exposición. Demostrar cómo influye el comportamiento de la penetración de iones cloruro en la corrosión de los aceros de refuerzo en dependencia de la condición de exposición a las cuales fueron sometidos los hormigones.

La Terminal de Cruceros llamada Sierra Maestra, se encuentra situada en la Bahía de la Habana, en pleno Centro Histórico de la capital cubana. Se trata de una edificación significativa de estilo Ecléctico inaugurada en el año 1914 que encerró a la Plaza de San Francisco quitándole en ese sitio el acceso al mar. Es el lugar más concurrido en cuanto a la llegada de los cruceros que viajan a la isla (Figura. 1).Los resultados obtenidos permitirán conocer si es necesario o no, proceder con los trabajos y esquemas de mantenimiento o reparación.

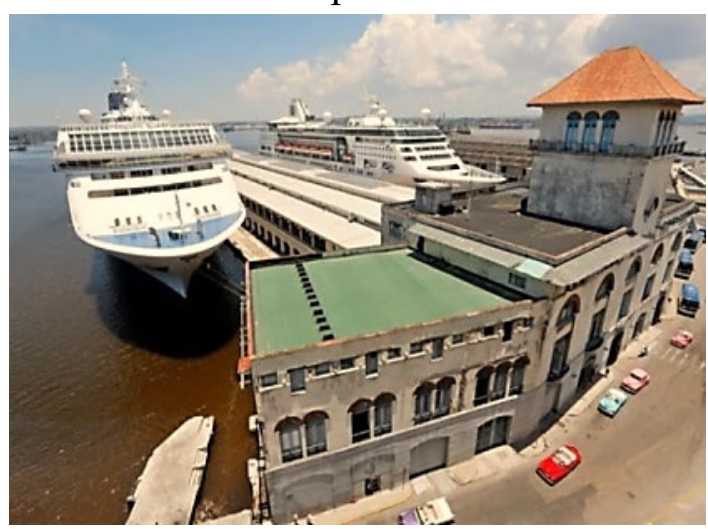

Figura.1. Vista de la terminal de cruceros en la bahía de la Habana. La estructura de hormigón armado arriba a la derecha es la que está siendo sometida a los trabajos de ampliación.

\section{PROCEDIMIENTO EXPERIMENTAL}

\subsection{Extracción de las probetas de hormigón simple}

Siete probetas de hormigón simple en forma cilíndrica es decir, sin aceros de refuerzo de $80 \mathrm{~mm}$ de diámetro y $300 \mathrm{~mm}$ de altura, fueron extraídas de 7 pilotes expuestos a diferentes condiciones de exposición frente al agua de mar de la bahía de la Habana (Figura. 2 a) y c) haciendo uso de una máquina de extracción de testigos de marca WE de fabricación china. El pilote 5 estuvo más sometido a las condiciones de marea y salpicadura, debido a que se encontró en la parte más exterior que sostiene la loza armada. El espesor de recubrimiento de hormigón usado en los pilotes durante la construcción de la bahía es alrededor de los 100 mm (Figura 2 a).
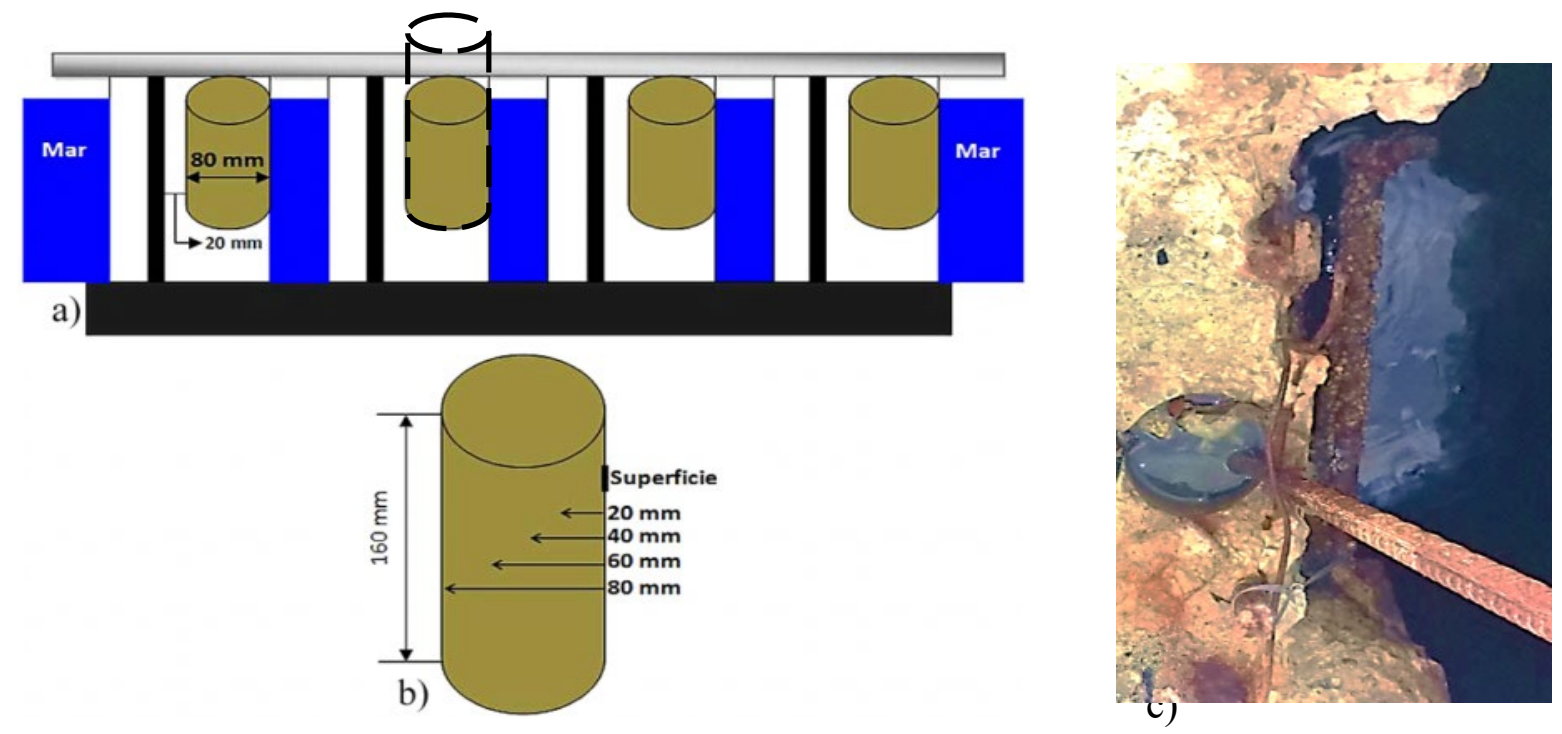
Figura.2. Esquemas y figura que muestran cómo fue realizada la toma de las probetas cilíndricas en la terminal, a) y c), así como sus dimensiones, b).

Posteriormente, las probetas fueron cortadas con una sierra de tungsteno de marca CUSHION CUT de fabricación estadounidense. De esta manera, las probetas cilíndricas quedaron con una dimensión de $80 \mathrm{~mm}$ diámetro por $160 \mathrm{~mm}$ de altura (Figura. $2 \mathrm{~b}$ ). Se trata de la zona donde la superficie de los pilotes estuvo en contacto con el agua de mar para la evaluación completa de la penetración de iones cloruro.

\subsection{Determinación de la concentración de iones cloruro totales y solubles a diferentes profundidades}

La penetración de iones cloruro, se realizó a partir de la toma de muestras de polvo en las probetas cilíndricas desde la superficie lateral hasta 20, 40, 60 y $80 \mathrm{~mm}$ de profundidad (Figura $2 \mathrm{~b}$ ). Se extrajeron para cada caso $40 \mathrm{~g}$ de muestras de polvo de las cuales, dos muestras de 10 gramos (20 g) fueron usados para la determinación de los dos valores de concentración de iones cloruro totales $\left(\boldsymbol{C} \boldsymbol{l}_{\boldsymbol{t}}^{-}\right)$y las otras dos muestras de 10 gramos para la determinación de los dos valores de concentración de iones cloruro solubles $\left(\boldsymbol{C l}_{\boldsymbol{s}}^{-}\right)$. La concentración de iones cloruro enlazados $\left(\boldsymbol{C l}_{\boldsymbol{e}}^{-}\right)$ fue determinada despejándolo de la conocida ecuación:

$$
C l_{t}^{-}=C l_{s}^{-}+C l_{e}^{-}
$$

Los dos valores de concentración de iones cloruro totales $\left(\boldsymbol{C l} \boldsymbol{l}_{\boldsymbol{t}}^{-}\right)$, solubles $\left(\boldsymbol{C l} \boldsymbol{l}_{\boldsymbol{s}}^{-}\right)$y enlazados $\left(\boldsymbol{C l} \boldsymbol{l}_{\boldsymbol{e}}^{-}\right)$ en porciento en masa de hormigón $(\% \mathrm{mh})$, fueron graficados en función de la profundidad de penetración con el propósito de demostrar el comportamiento de la penetración de iones cloruro en las siete probetas extraídas.

La extracción de las muestras de polvo se realizó usando un taladro de banco de fabricación inglesa de marca ROCWELL DELTA con una escala para la medición de la profundidad de extracción. Las probetas fueron colocada de forma vertical y bien sujetadas en un martillo de banco para garantizar la penetración perpendicular de la barrena sin problema alguno, exactamente en la misma dirección de la penetración de los iones cloruro desde el mar hacia el interior de los pilotes de hormigón armado.

Primeramente, la extracción de las muestras de polvo en la superficie fue ejecutada con una espátula muy fina hasta una profundidad no superior a $5 \mathrm{~mm}$. Para las diferentes profundidades se empleó una barrena de tungsteno con un diámetro de 18 mm (RILEM TC 178-TMC:2013). De acuerdo con la información obtenida por la compañía que centra la ejecución de los trabajos de rehabilitación, el hormigón usado en la construcción de los pilotes, su árido grueso presentó un tamaño nominal de $19 \mathrm{~mm}$. La extracción fue ejecutada siempre en la parte central de cada probeta y las muestras de polvo fueron almacenadas en bolsitas de nylon muy bien identificadas. Antes de ejecutar la penetración en cada muestra y profundidad, siempre fue realizada una limpieza de la barrena con un cepillo dental, con el fin de tener una mayor exactitud en las determinaciones.

La determinación de la concentración de iones cloruro totales y libres fue realizadasegún las especificaciones establecidas en la normativa (ASTM C-1218/M: 2008) haciendo uso del método químico-analítico de valoración volumétrica, donde el reactivo valorante fue el $\mathrm{AgNO}_{3}$.

\section{RESULTADOS Y DISCUSIÓN}

3.1. Comportamiento de la penetración de iones cloruro en los pilotes de la terminal Es de notar según el comportamiento (Figura 3 a), b), c), d), e) f) y g), la presencia de un perfil de penetración solamente en la probeta 5 a partir de $20 \mathrm{~mm}$ hasta $60 \mathrm{~mm}$ de profundidad para los $C \boldsymbol{l}_{\boldsymbol{t}}^{-}$ (rojo y negro) y $\boldsymbol{C l} \boldsymbol{l}_{\boldsymbol{s}}^{-}$(verde y azul) (Figura 3 e). Esto se debe, a que se trató del pilote que estuvo sometido en ocasiones a la condición de marea y salpicaduras, a la hora de entrar y atracar los cruceros en la terminal muy frecuente en la temporada turística en Cuba (Noviembre-Marzo), así 
como de grandes buques para las operaciones de carga y descarga en la bahía. De esta forma, puede originarse la difusividad aparente de los iones cloruro a partir de cierta profundidad debido a una mayor variación de la temperatura y la humedad existente en el interior del hormigón armado hasta ese espesor de recubrimiento de $20 \mathrm{~mm}$.

a)

b)

c)

d)

e)

f)

g)

XVI Congreso Latinoamericano de Patología de la Construcción 
Figura. 3. Comportamiento de la penetración de iones cloruro a diferentes profundidades en las siete probetas de hormigón.

Se trata del conocido fenómeno de la cristalización de las sales, debido precisamente al incremento de la temperatura a partir del ciclo de humectación y secado a escasos espesores de recubrimiento de hormigón. Como bien se conoce, este comportamiento ha sido más típico en los elementos de las estructuras (vigas y columnas) y probetas de hormigón armado sometidos al impacto del ambiente agresivo en zonas costeras caracterizadas por una variación considerable de la temperatura y la humedad relativa de la atmósfera y deposiciones elevadas de sales de iones cloruro (Castro, 1998; Corvo, et al 2008; Meira, et al 2010). Esta variación, tiende a ocurrir en el interior del hormigón armado hasta ciertos espesores de recubrimiento $(20 \mathrm{~mm})$ principalmente en hormigones porosos. La agresividad corrosiva de la atmósfera ha resultado elevada (C4), muy elevada (C5) y extrema (CX). Ha ocurrido también en pilotes que sostienen la loza armada de puentes, expuestos al mar abierto en zonas de intenso rompiente de las olas, donde se originan grandes salpicaduras y zonas de marea. Para todos los casos, se ha demostrado una corrosión muy acelerada en los aceros de refuerzo provocando la formación de fisuras y grietas en el espesor de recubrimiento de hormigón, facilitando una mayor penetración de los agentes agresivos como son las sales de iones cloruro y el oxígeno (Castro, 1998).

La penetración de las sales de iones cloruro en el resto de las probetas presentaron un comportamiento muy variable (Figura 3 a), b), c), d), e) y g). En ningún momento se ilustra el comportamiento difusivo de los iones cloruro desde la superficie o partir de $20 \mathrm{~mm}$ hasta $80 \mathrm{~mm}$ de profundidad de penetración, característico en pilotes de hormigón armado y probetas sumergidas totalmente en agua de mar o en disoluciones de $\mathrm{NaCl}$ entre 3-5\% bajo condiciones de laboratorio. Este comportamiento se debe a la permanencia constante del agua, factor imprescindible para que se origine el fenómeno de la difusión de los iones cloruro en el hormigón armado.

Por otra parte, es de observar como en las probetas desde la 1 hasta la 5 (Figuras 3 a), b), c), d), e), la $\boldsymbol{C l} \boldsymbol{l}_{\boldsymbol{e}}^{-}$resultó en ocasiones superior a la $\boldsymbol{C \boldsymbol { l } _ { \boldsymbol { s } } ^ { - }}$ desde la superficie hasta las diferentes profundidades. Incluso en la probeta 5 (Figura 3 e), es de apreciar, como la $\boldsymbol{C l}_{\boldsymbol{e}}^{-}$(colores Cyan y Magenta) presentó un comportamiento similar a la $\boldsymbol{C l}_{\boldsymbol{t}}^{-}$. Este comportamiento, ha resultado inusual a la hora de estudiar la penetración de iones cloruro en el hormigón armado sometido principalmente a la condición de inmersión completa en agua de mar o en soluciones salinas bajo condiciones de laboratorio.

El hecho de que la $\boldsymbol{C l}_{\boldsymbol{e}}^{-}$sea mayor que $\boldsymbol{C l}_{\boldsymbol{s}}^{-}$; pudiera deberse a que el hormigón usado en la construcción de los pilotes que sostienen la loza armada de la Terminal de Cruceros de la Bahía de la Habana fue elaborado con arena de mar. Aunque haya sido sometida a procesos de lavado muy eficiente, siempre los iones cloruro permanecen y usando además agua de mar en el amasado. Esto pudiera ser la condición que justifica la no aparición de un comportamiento difusivo es decir, la fuente de procedencia de los iones cloruro hacia interior del hormigón no es solo el agua de mar. De lo contrario, las concentraciones de iones cloruro, principalmente la $\boldsymbol{C l}_{\boldsymbol{e}}^{-}$no hubiesen resultado tan elevadas.

Los pilotes existentes en la terminal no mostraron signos de fisuración y agrietamiento alguno, como un indicador muy confiable en el desarrollo de la corrosión en los aceros de refuerzo. La ocurrencia insignificante de la corrosión en los acero de refuerzo se debe a dos cuestiones fundamentales. Primero, a la baja solubilidad del oxígeno en el agua de mar. Además, para una mayor concentración de sales, el oxígeno es menos soluble aun en este caso en el agua de mar. Esto hace que no llegue una concentración crítica de oxígeno a la superficie de los aceros de refuerzo capaz de desarrollar el fenómeno de la corrosión electroquímica a pesar de que la concentración de iones cloruro sobre todo totales y solubles resultó muy elevada. De ahí, a que los elementos de 
hormigón armado sometidos a inmersión total en agua de mar sean durables garantizando plazos elevados de vida útil a la estructura. Las elevadas concentraciones de iones cloruro pudiera ser una condición necesaria pero no suficiente en el origen de la corrosión.

La observación visual de algunos de los aceros de refuerzo extraído de las probetas, permite confirmar la casi no ocurrencia del fenómeno de la corrosión (Figura 4). Es de notar que estos aceros de refuerzo presentaron diferentes conformaciones. Las costras observadas de color carmelita y de espesores muy bajos, obedecen a la formación de sales de cloruro de hierro, sobre todo la de $\mathrm{FeCl}_{3}$. Esta sal,originada de la ruptura de la capa pasiva en el proceso electroquímico del fenómeno de la corrosión debido a la influencia de los iones cloruro, es la que tiende a reaccionar con el hidróxido $\left(\mathrm{OH}^{-}\right)$, originado en el proceso de reducción catódica del oxígeno a partir de la conocida reacción: $\boldsymbol{O}_{2}+\mathbf{2} \boldsymbol{H}_{2} \boldsymbol{O}+\mathbf{4} \boldsymbol{e}^{-} \rightarrow \mathbf{4} \boldsymbol{O} \boldsymbol{H}^{-}$. Al no existir una reducción considerable del oxígeno debido a su solubilidad muy baja y más en agua de mar, no se garantiza una concentración crítica del ion hidróxido capaz de reaccionar con la sal de $\mathrm{FeCl}_{3}$ para de esta manera originarse los productos de corrosión correspondientes (Troconis et al 1997).

Figura. 4. Observación

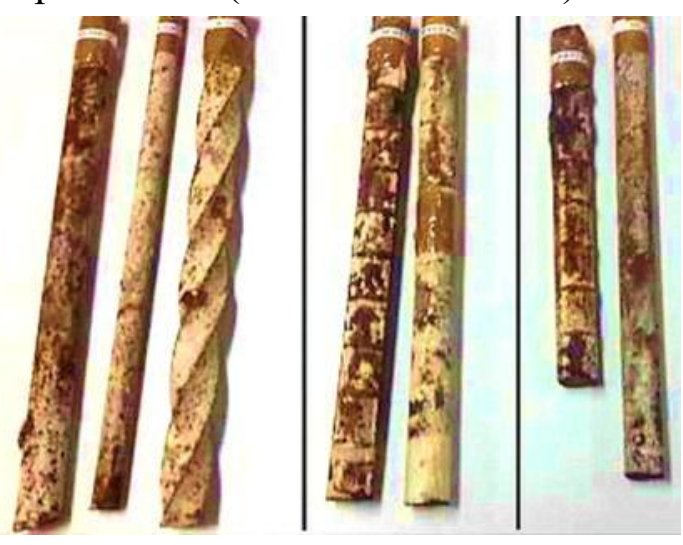

refuerzos.

visual de los aceros de

Todo lo contrario resulta en los hormigones expuestos a categorías elevadas de agresividad corrosiva de la atmósfera o sometidos a ensayos acelerados bajo condiciones reales de exposición, donde existe una mayor penetración del oxígeno debido al incremento de la temperatura de la atmósfera y en el interior del hormigón, sobre todo en hormigones muy porosos. De esta manera, llega a los aceros de refuerzo una concentración mayor de oxígeno, donde la corrosión se inicia a partir de la ruptura de la capa pasiva originada por los iones cloruro principalmente los solubles.El hormigón usado en la construcción de los pilotes presentó una resistencia a la compresión promedio alrededor de $20 \mathrm{MPa}$ y un porcentaje de porosidad capilar efectiva entre $15-20 \%$. Se trata de un hormigón de moderada resistencia mecánica y porosidad elevada. Sin embargo, todo parece indicar que puede ser usado en la construcción de pilotes que sostienen la loza armada, siempre y cuando estén sometidos a la condición de inmersión total en agua de mar en bahías. Como bien se conoce, las aguas de las bahías se caracterizan por ser tranquilas es decir, sin la presencia de las olas intensas. Las olas al impactar en los pilotes incluso hasta en la loza armada de los puentes expuestos al mar abierto originan las condiciones de marea y salpicadura. Estos tipos de hormigones expuestos a dichas condiciones, no deben garantizar una durabilidad y vida útil elevada.

Es de notar, como la penetración de los iones cloruro totales $\left(\boldsymbol{C l}_{\boldsymbol{t}}^{-}\right)$(Figura 5 a) y solubles $\left(\boldsymbol{C l}_{\boldsymbol{s}}^{-}\right.$) (Figura $5 \mathrm{~b}$ ), presentaron un comportamiento aparentemente difusivo en probetas de hormigón armado (200 x $200 \times 200 \mathrm{~mm}$ ) para las tres relaciones agua/cemento 0,4; 0,5 y 0,6 (espesores de recubrimiento 20 y $40 \mathrm{~mm}$ ) expuestas durante tres años de exposición en un sitio a $20 \mathrm{~m}$ desde la línea costera en el litoral norte de La Habana. La deposición promedio anual de sales de iones cloruro fue de $770 \mathrm{mg} / \mathrm{m}^{2} \mathrm{~d}$ determinada por el captador del paño seco y de $1848 \mathrm{mg} / \mathrm{m}^{2} \mathrm{~d}$ estimada por el captador de la vela húmeda. A tal punto que, en los meses más invernales de Cuba 
(Noviembre-Marzo), la deposición mensual determinada en el sitio por el captador del paño seco, resultó mayor que $1000 \mathrm{mg} / \mathrm{m}^{2} \mathrm{~d}$. Para estas condiciones se garantiza una agresividad corrosiva extrema (CX). Se trata de una de las zonas más agresivas a nivel mundial (Castañeda, et al 2019).
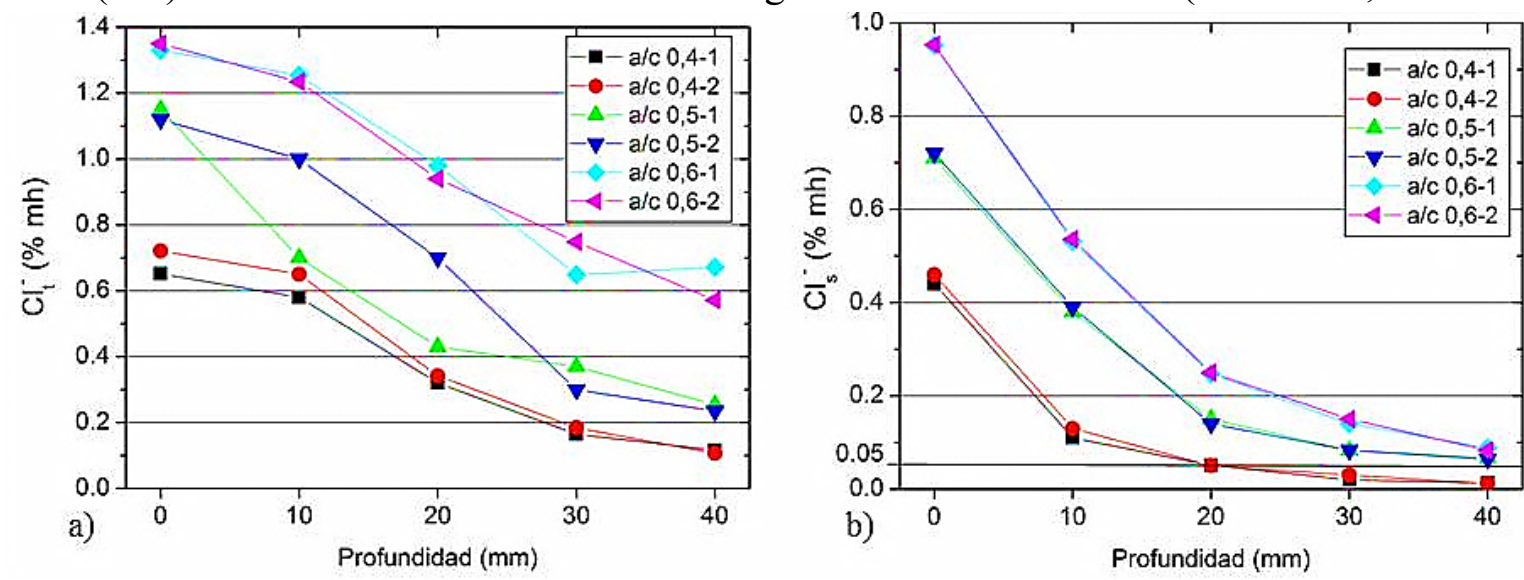

Figura.5. Comportamiento de la penetración de iones cloruro en el sitio costero de La Habana.

Las características de las tres proporciones de las mezclas de hormigón usadas en la elaboración de las probetas son mostradas (Tabla 1). Las probetas fueron elaboradas a partir del método de dosificación del porcentaje mínimo de vacío. Los ajustes correspondientes para cada dosificación de materiales fueron realizados para $1 \mathrm{~m}^{3}$ de volumen neto de hormigón. Cuando las tres mezclas de hormigón fueron diseñadas, el agregado fino (arena de río, finura $3 \mathrm{~mm}$ ) y el árido grueso calizo (tamaño nominal $19 \mathrm{~mm}$ ) fueron pesados hasta peso constante. Por tanto, cada dosificación fue diferente al variar la relación agua/cemento $(\mathrm{a} / \mathrm{c})$. El tiempo de curado en las probetas fue de 28 días a inmersión total en agua, donde su temperatura fue $25^{\circ} \mathrm{C}$.

Tabla. 1. Proporciones de mezclas de los hormigones usados en el estudio.

\begin{tabular}{|c|c|c|c|c|}
\hline $\mathrm{a} / \mathrm{c}$ & $\begin{array}{c}\text { Cemento } \\
\text { OPC } \\
\left(\mathrm{kg} / \mathrm{m}^{3}\right)\end{array}$ & $\begin{array}{c}\text { Agua } \\
\text { potable } \\
\left(\mathrm{m}^{3}\right)\end{array}$ & $\begin{array}{c}\text { Aditivo } \\
\text { superplastificante } \\
\left(\mathrm{m}^{3}\right)\end{array}$ & $\begin{array}{c}\text { Asentamiento } \\
(\mathrm{cm})\end{array}$ \\
\hline \multirow{2nnnyy}{*}{365} & 148 & 1,7 & 15 \\
\cline { 1 - 3 } & \multirow{2}{*}{365} & 186 & 1,5 & 17 \\
\cline { 1 - 1 } 0,6 & 222 & 1,0 & 18 \\
\hline
\end{tabular}

El comportamiento aparentemente difusivo permite demostrar que, los hormigones de las probetas nos fueron elaborados usando arena y agua de mar para su amasado es decir, los iones cloruro provienen desde el exterior. La fuente de procedencia de los iones cloruro es el mar transportados en el aerosol marino. Caso contrario al hormigón usado en los pilotes, donde existe la posibilidad que los iones cloruro además de proceder del mar, ya se encontraban presentes en el interior del hormigón. Por otra parte, los valores promedios mensuales y anuales de humedad relativa resultaron muy elevados $\left(>80 \%\right.$ y T $\left.=25{ }^{\circ} \mathrm{C}\right)$ producto al fuerte impacto permanente del aerosol marino en el sitio de exposición durante los tres años de estudio (Figura 5 a) y b). Esto conlleva a la presencia de agua casi permanente en los poros del hormigón, principalmente desde la superficie hasta $10 \mathrm{~mm}$ de espesor de recubrimiento como condición necesaria para que se origine el carácter aparentemente difusivo como mecanismo de penetración de los iones cloruro. Como bien se conoce, el hormigón gana el agua más rápido de lo que la pierde y más expuestos a climas tropicales costeros de elevada agresividad corrosiva de la atmósfera.

Debido a estas condiciones, un comportamiento difusivo aparente de la penetración de iones cloruro totales (\% en masa de hormigón) desde la superficie hasta una profundidad de $40 \mathrm{~mm}$ de espesor de recubrimiento de hormigón, fue demostrado en los tímpanos en forma de pantalones 
que sostienen un edificio de hormigón armado terminado de construir a finales del año 1973 (Carvajal, et al 2011). La estructura se encuentra expuesta a una agresividad corrosiva de la atmósfera extrema (CX) localizada también en el litoral norte del malecón de la Habana, Cuba a $20 \mathrm{~m}$ desde la línea costera. Las mayores concentraciones fueron obtenidas en la zona menos impactada por la lluvia (Figura. 6). Los ensayos de penetración fueron ejecutados 30 años después de construida la obra (2003). Las muestras de polvo fueron extraídas usando también un taladro percutor (20 g para dos muestras en $10 \mathrm{~g}$ de cloruro totales), cumpliéndose la condición de que el diámetro de la barrena $(18 \mathrm{~mm})$ como sucedió en las probetas y en los pilotes. Se trató de un hormigón muy denso y compacto $(\mathrm{a} / \mathrm{c}=0,4)$, con una resistencia a la compresión promedio de 50 MPa y un contenido de cemento de $450 \mathrm{~kg} / \mathrm{m}^{3}$.

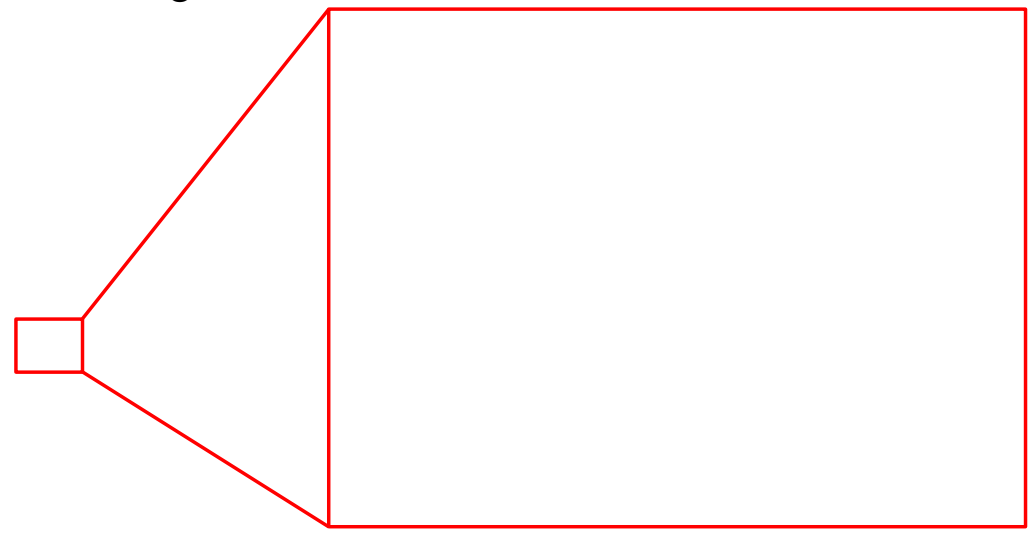

Figura.6. Comportamiento de la penetración de iones cloruro en uno de lostímpanos del edificio ubicado en el litoral norte costero de la Habana, Cuba.

De acuerdo con la observación visual de las probetas, es de notar a simple vista la formación de grietas longitudinales paralelas a los aceros de refuerzo debido a la fuerza de expansión que ejercen los productos de corrosión (óxidos metálicos) de forma perpendicular a la superficie, siendo más significativo para un espesor de recubrimiento de $20 \mathrm{~mm}$ (Figura $7 \mathrm{a} \mathrm{y} \mathrm{b).}$

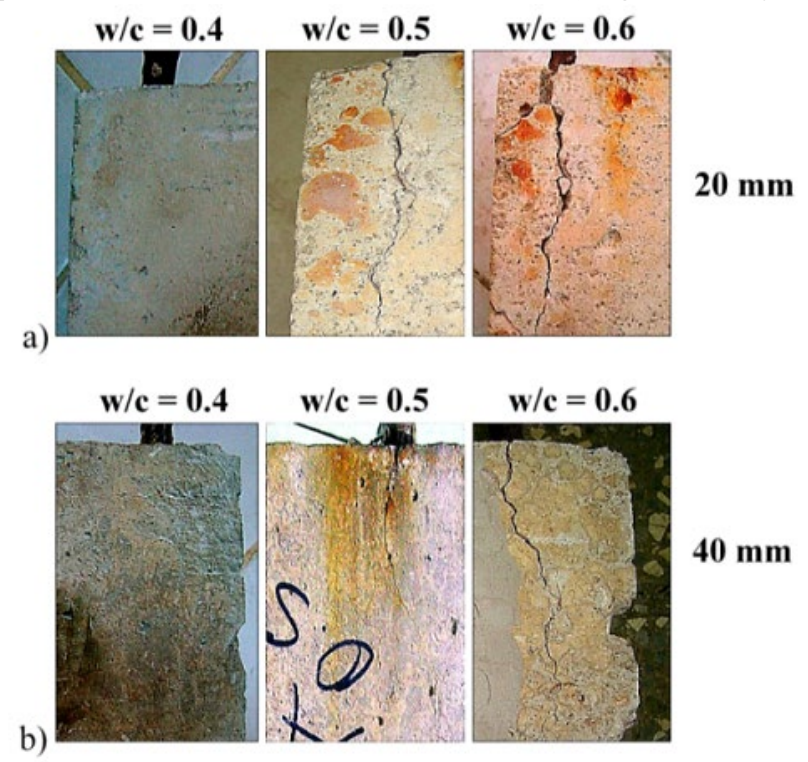

Figura. 7. Observación visual de las probetas para ambos espesores de recubrimiento de hormigón, 20 y $40 \mathrm{~mm}$.

Se trata de las condiciones de diseño más usadas en la elaboración del hormigón en Cuba con una resistencia a la compresión promedio de 36,30 y $25 \mathrm{MPa}$ y porcentaje de porosidad capilar de 8 , 14 y $20 \%$ para las relaciones agua/cemento 0,$4 ; 0,5$ y 0,6 respectivamente. Se confirma que, la fuerza de expansión (MPa) de los productos de corrosión una vez desarrollado el fenómeno de la 
corrosión atmosférica en los aceros de refuerzo, es muy superior a la resistencia a la tracción de las probetas. Condición necesaria para la formación de fisuras y grietas, lo cual origina una mayor penetración de los agentes agresivos acelerando de manera considerable el fenómeno.

Sin embargo y como aspecto curioso, es de notar como estos mismos hormigones pero en probetas de diferentes dimensiones $(315 \times 80 \times 80 \mathrm{~mm}$, espesor de recubrimiento de hormigón $=20 \mathrm{~mm})$ y sometidos a ensayos acelerados bajo condiciones reales de exposición a la atmósfera, a partir de la aplicación de un spray salino de $\mathrm{NaCl}$ al 3\% diariamente en horas de la mañana, el comportamiento de la penetración de iones cloruro resultó diferente a los referidos anteriormente y también al de los pilotes sumergidos totalmente en agua de mar (Figura 7 a). Se aprecia además, la clara existencia del proceso de humectación y secado (cristalización de la sal).
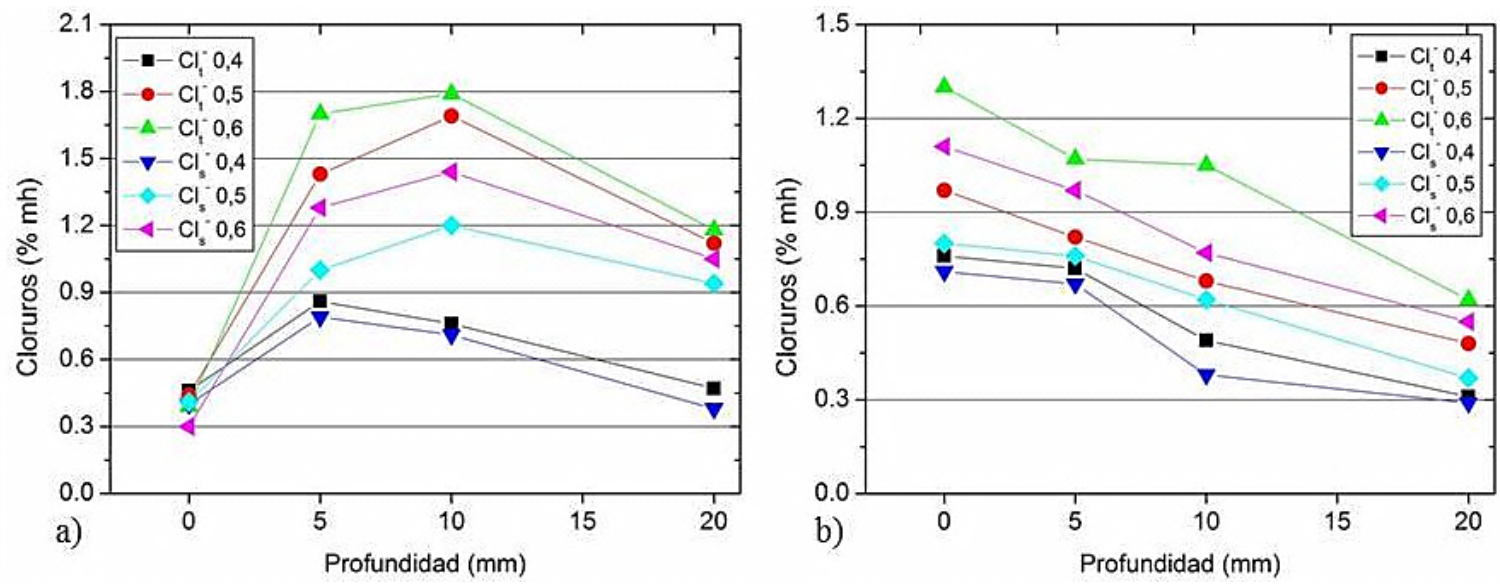

Figura. 7. Comportamiento de la penetración de iones cloruro, a) condiciones reales de exposición a la atmósfera, b) inmersión total en la disolución salina al 3\%.

Sin embargo, para la condición de inmersión total en la misma disolución salina simulando agua de mar, es de apreciar un comportamiento aparentemente difusivo para las tres relaciones agua/cemento 0,4; 0,5 y 0,6 (Figura 7 b), como sucedió en las probetas anteriores, en el edificio, pero no en los pilotes de la terminal (Castañeda, et al 2005). Se confirma que, al no ser un hormigón elaborado con arena y agua de mar, es de esperar un comportamiento difusivo en cuanto a la penetración de iones cloruro, ya sea desde la superficie o partir de una cierta profundidad precedido del ciclo de humectación y secado.

La aplicación del spray salino de $\mathrm{NaCl}$ al 3\% en el tiempo referido bajo condiciones reales de exposición (tres años), representa una deposición promedio anual de iones cloruro igual a $850 \mathrm{mg} / \mathrm{m}^{2} \mathrm{~d}$ determinada por el captador del paño seco. De esta forma, se está en presencia de una agresividad corrosiva o corrosividad de la atmósfera extrema $(\mathrm{CX})$, típica de zonas costeras a corta distancia de la zona del rompiente de las olas (Corvo, et al 2005). Se observa, como la difusividad comenzó y el ciclo de humectación y secado finalizó a partir de $10 \mathrm{~mm}$ de espesor de recubrimiento para los $\boldsymbol{C l}_{\boldsymbol{t}}^{-}$y $\boldsymbol{C \boldsymbol { s } _ { \boldsymbol { s } } ^ { - }}$ en las probetas de relaciones agua/cemento 0,5 y 0,6. No siendo así en la probeta de relación agua/cemento 0,4 donde la difusividad se inició y el ciclo culminó a partir de $5 \mathrm{~mm}$ (Figura 7 a). Es evidente que el ciclo finaliza a una mayor profundidad en los hormigones de relación agua/cemento 0,5 y 0,6 caracterizados por una porosidad elevada $(>10 \%)$.

La observación visual, confirma la influencia de la agresividad corrosiva de la atmósfera extrema en las probetas de hormigón armado expuestas en la estación de ensayo. Es de notar también, la presencia del agrietamiento en las probetas de relaciones agua/cemento 0,5 y 0,6 sometidas al spray 
salino, así como una pequeña fisura longitudinal en la probeta de relación agua/cemento 0,6 sometida a inmersión total en la disolución salina (Figura 8.)

La diferencia en cuanto al mecanismo de penetración de iones cloruro hacia el interior del hormigón, comparada con la condición de exposición en el sitio costero referido anteriormente y el edificio, pudiera deberse a la forma de aplicación del spray salino.

$$
\text { Spray salino Inmersión }
$$

Figura. 8. Observación visual de las probetas colocadas en la estación de ensayo y a inmersión en la disolución salina de $\mathrm{NaCl}$ al 3\%.

Al aplicarse el mismo en horas de la mañana diariamente, también a valores elevados de humedad relativa en la estación de ensayo donde fueron colocadas las probetas $\left(\sim 80 \%\right.$ y $\left.\mathrm{T}=25{ }^{0} \mathrm{C}\right)$, era de notar una absorción muy rápida de la disolución salina en las superficies principalmente en las probetas de relaciones agua/cemento 0,5 y 0,6 , además de un secado acelerado debido a la fuerte intensidad de la radiación solar. Esta aplicación al realizarse solo en horas de la mañana, tiene un carácter menos permanente en comparación con el impacto del aerosol marino en el litoral norte de La Habana. El impacto del aerosol marino es casi constante. Solo en el primer año de exposición de las probetas en el sitio costero, penetraron desde la dirección norte 17 frentes fríos en la etapa invernal. En la etapa de verano (Abril-Septiembre) a pesar de la no ocurrencia de la entrada de los frentes, la deposición mensual estuvo entre 250-500 mg/ $\mathrm{m}^{2} \mathrm{~d}$ (Castañeda, et al 2018). De ahí al embate permanente del aerosol marino depositándose los iones cloruro sobre las probetas de hormigón armado en forma de disolución salina durante los tres años.

\section{CONCLUSIONES}

Se realizó un estudio sobre el comportamiento de la penetración de iones cloruro en los pilotes de hormigón armado expuestos totalmente a inmersión en agua de mar, que sostienen la loza armada de la Terminal de Cruceros de la bahía de la Habana, Cuba y su comparación con otros tipos de hormigones sometidos a otras condiciones de exposición. Los resultados permitieron arribar a las siguientes conclusiones.

1. El comportamiento de la penetración de iones cloruro totales y solubles en los pilotes de la terminal, resultó totalmente diferente en comparación con otros tipos de hormigones expuestos a la condición de exposición a la atmósfera bajo condiciones de muy elevada (C5) y extrema (CX) agresividad corrosiva de la atmósfera. Esta diferencia en cuanto a la obtención de un comportamiento no difusivo en los pilotes, pudiera deberse a que el hormigón fue construido con arena y agua de mar. De ahí, a que la concentración de iones cloruro totales, solubles y enlazado haya resultado muy elevada.

2. Se demuestra que, si los hormigones son elaborados sin arena y agua de mar, existe la posibilidad de un comportamiento difusivo aparente en cuanto al mecanismo de penetración 
de iones cloruro, procedentes desde el exterior, independiente de la condición de exposición.

3. Se confirma que, un hormigón armado de moderada calidad elaborado con arena y agua de mar, con una elevada concentración de iones cloruro, puede ser usado en la construcción de pilotes que sostienen la loza armada en una bahía, siempre y cuando estén sometidos a la condición de inmersión total en el agua de mar y sin zonas de marea y salpicaduras significativas. Para este caso, las concentraciones elevadas de iones cloruro resultan en una condición necesaria pero no suficiente en el origen y desarrollo del fenómeno de la corrosión en los aceros de refuerzo. Este resultado fue confirmado con la observación visual de los aceros de refuerzo embebidos en los pilotes y permitió prácticamente la no ejecución de trabajos de reparación siendo muy factible económicamente.

4. El mecanismo de penetración de iones cloruro depende de la calidad del hormigón y de la condición de exposición a la cual fue expuesto. El comportamiento basado en la difusión aparente, no solo a partir de una espesor de recubrimiento, sino también desde la superficie, conducen a un intenso deterioro en el hormigón armado, sometido principalmente a categorías elevada de agresividad corrosiva de la atmósfera en zonas costeras de elevado potencial constructivo.

\section{AGRADECIMIENTOS}

Agradecer a la Dirección de Desarrollos Tecnológico de Ministerio de la Construcción y la Empresa Nacional de Investigaciones Aplicadas de Cuba, por el apoyo logístico y financiero en la ejecución del estudio.

\section{REFERENCIAS}

Angst, U., Elsener, B., Larsen, C. K and Vennesland, Ø. (2009), Critical chloride content in reinforced concrete - A review. Cement and Concrete Researchs. 39(1): 1122-1138.

Angst, U.,Elsener, B., Larsen, C, K., Vennesland, Ø. (2011), Chloride induced reinforcement corrosion: Electrochemical monitoring of initiation stage and chloride threshold values. Revista Corrosion Science. 53, pp. 1451-1464.

El Fattah, A, Abd., Al-Duais, I., Riding, Kyle., Thomas, Michael. (2019), Field evaluation of corrosion mitigation on reinforced concrete in marine exposure conditions. Construction and Building Materials 165, 663-674.

ASTM International. (2008). ASTM C-1218/M (2008).Standard Test Method for Water-Soluble Chloride in Mortar and Concrete, Barr Harbor Drive, USA.

Castañeda, A.,Corvo, F and O' Reilly,V. (2003), Comparación entre el pronóstico de corrosión basado en la medición de potenciales y la determinación de la velocidad de corrosión de la barra de refuerzo mediante técnicas electroquímicas. Revista Materiales de Construcción, CSIC.53, (271-272): $155-164$.

Castañeda, A., Días,Nelson., González,E., Martínez,M., Corvo, F. (2005), Influencia de la penetración de iones cloruros en el hormigón armado a diferentes relaciones agua/cemento y condiciones de exposición. REVISTA CENIC, EBSCO, CIENCIAS QUÍMICAS, Vol. 36, No. ESPECIAL, CIUDAD DE LA HABANA, CUBA.

Castañeda, A.,Corvo, F., Howland, J.J and Pérez, T. (2013), Corrosion of steelreinforced concrete in tropical coastalatmosphere of Havana City.Revista Química Nova, Brasil. 36 (2): 220-229.

Castañeda, A.,Corvo, F., Howland, J. J and Marrero R. (2018), Penetration of marine aerosol in a tropical coastal City: Havana.Revista Atmósfera.31 (1): 87-104.https://doi: 10.20937/ATM.2018.31.01.06. 
Castañeda, A.,Corvo, F., Howland, J. J., Marrero, R and Fernández A.(2019),Atmospheric Corrosion Behaviors of Reinforcement Steel in Reinforced Concrete in a Coastal City as Havana,Cuba.Springer Nature Switzerland AG 2020 F. Martirena et al. (eds.), Proceedings of the International Conference of SustainableProduction and Use of Cement and Concrete, RILEM Book22 (1): 83-291.

Carvajal, M., Vera, R., Corvo,FandCastañeda,A. (2011),Diagnosis and rehabilitation of real reinforced concrete structures in coastal areas. CorrosionEngineering, Science And Technology. 47 (1): 1-9. https://doi.org/10.1179/1743278211Y.0000000018.

Castro, P. (1998), "Corrosión en Estructuras de Concreto Armado. Teoría, Inspección, Diagnóstico, Vida Útil y Reparaciones”. Instituto Mexicano del Cemento y del Concreto, A.C., Mérida.

Corvo,F., Minotas, J., Delgado, J and Arroyave, C. (2005), Changes in atmospheric corrosion rate caused by chloride ions depending on rain regime, Corrosion Science, 47 (1): 883892.https://doi:10.1016/j.corsci.2003.09.014.

Corvo, F., Pérez, T., Dzib, L. R., Martín, Y., Castañeda, A., González, E and Pérez, J. (2008), Outdoor-Indoor corrosion of metals in tropical coastalatmospheres.Corrosion Science.50(1):220 - 230.https://doi:10.1016/j.corsci.2007.06.011.

Helene, P. (2003), "Manual de Rehabilitación de Estructuras de Hormigón. Reparación, Refuerzo y Protección”. EditorialCyTED, Sao Paulo, Brasil.

Meira, G.R., Andrade, C., Padaratz, I.J., Alonso, C andBorba J.C. Jr.(2007), Chloride penetrations into concrete structure in the marine atmosphere zone. Relationship between deposition of chloride on the wet candle and chloride accumulated into concrete. Cement and Concrete Composite.29 (1): pp. 667-676.doi:10.1016/j.cemconcomp.2010.03.002.

Meira, G.R., Andrade, C., Alonso, C.,Padaratz, I.J., and Borba J.C. Jr. (2008),Modelling sea-salt transport and deposition in marine atmosphere zone - A tool for corrosion studies. Corrosion Science.50 (1): 2724-2731.https://doi:10.1016/j.corsci.2008.06.028.

Meira, G.R., Andrade, C., Alonso.,Borba J.C. Jr and Padilha. M. (2010),Durability of concrete structure in marine atmosphere zone.The use of chloride deposition rate on wet candle as an environment indicator.Cement and Concrete Composites.32 (2): 427435.https://10.1016/j.cemconcomp.2010.03.002.

Norma ISO-9223 (2012), Corrosion of metals and alloys.Corrosivity of atmospheres.Classification, determination and estimation.

Pradhan, B and Bhattacharjee, B. (2011), Rebar corrosion in chloride environment.Construction and Building of Materials. $25 \quad$ (11): $\quad$ pp. $\quad$ 25652575.https://doi.org/10.1016/j.conbuildmat.2010.11.099.

RILEM TC 178-TMC: Testing and modeling chloride penetration in concrete. Methods for obtaining dust samples by means of grinding concrete in order to determine the chloride concentration profile, Materials and Structures 46 (2013) 337-344.http://10.1617/s11527-0129968-1.

Trocónis, O., Manual de inspección, evaluación y diagnóstico de corrosión en estructuras de hormigón armado. CYTED, Red Temática XV. B. Durabilidad de la armadura, Río de Janeiro, Brasil, 1997.

Trocónis, O., Duracon Collaboration. (2007).Effect of the marine environment on reinforced concrete durability in Iberoamerican countries: DURACON project/CYTED. Corrosion Science. 49 (7): 2832-2843. https://doi.org/10.1016/j.corsci.2007.02.009. 
Xianming,S.,Ning,X.,Keith, F andJing, G. (2012),Durability of steel reinforced concrete inchloride environments: An overview. Construction and Building of Maters. 37 (2): pp. 3640.https://doi.org/10.1016/j.conbuildmat.2011.12.038 